\title{
Proposal for a spintronic femto-Tesla magnetic field sensor
}

\author{
S. Bandyopadhyay ${ }^{a}$ and M. Cahay $^{b}$ \\ ${ }^{a}$ Department of Electrical and Computer Engineering, Virginia Commonwealth \\ University, Richmond, VA 23284, USA \\ ${ }^{\mathrm{b}}$ Department of Electrical and Computer Engineering and Computer Science, \\ University of Cincinnati, OH 45221, USA
}

\begin{abstract}
We propose a spintronic magnetic field sensor, fashioned out of quantum wires, which may be capable of detecting very weak magnetic fields with a sensitivity of $\sim 1 \mathrm{fT} / \sqrt{H z}$ at a temperature of $4.2 \mathrm{~K}$, and $\sim 80 \mathrm{fT} / \sqrt{H z}$ at room temperature. Such sensors have commercial applications in magnetometry, quantum computing, solid state nuclear magnetic resonance, magneto-encephalography, and military applications in weapon detection.
\end{abstract}

Key words: spintronics, magnetic sensors, spin orbit interaction

PACS: 85.75.Hh, 72.25.Dc, 71.70.Ej

Preprint submitted to Elsevier Science

9 November 2018 
There is considerable interest in devising magnetic field sensors capable of detecting weak dc and ac magnetic fields. In this paper, we describe a novel concept for realizing such a device utilizing spin orbit coupling effects in a quantum wire.

Consider a semiconductor quantum wire with weak (or non-existent) Dresselhaus spin orbit interaction [1], but a strong Rashba spin orbit interaction [2] caused by an external transverse electric field. The Dresselhaus interaction accrues from bulk inversion asymmetry and is therefore virtually non-existent in centro-symmetric crystals, whereas the Rashba interaction arises from structural inversion asymmetry and hence can be made large by applying a high symmetry breaking transverse electric field. We will assume that the wire is along the x-direction and the external electric field inducing the Rashba effect is along the y-direction (see Fig. 1).

This device is now brought into contact with the external magnetic field to be detected, and oriented such that the field is directed along the wire axis (i.e. $\mathrm{x}$-axis). Assuming that the field has a magnetic flux density $B$, the effective mass Hamiltonian for the wire, in the Landau gauge $\mathbf{A}=(0,-B z, 0)$, can be written as

$$
\begin{aligned}
H= & \left(p_{x}^{2}+p_{y}^{2}+p_{z}^{2}\right) /\left(2 m^{*}\right)+\left(e B z p_{y}\right) / m^{*}+\left(e^{2} B^{2} z^{2}\right) /\left(2 m^{*}\right)-(g / 2) \mu_{B} B \sigma_{x} \\
& +V_{y}(y)+V_{z}(z)+\eta\left[\left(p_{x} / \hbar\right) \sigma_{z}-\left(p_{z} / \hbar\right) \sigma_{x}\right]
\end{aligned}
$$

where $g$ is the Landè g-factor, $\mu_{B}$ is the Bohr magneton, $V_{y}(y)$ and $V_{z}(z)$ are the confining potentials along the $\mathrm{y}$ - and z-directions, $\sigma$-s are the Pauli spin matrices, and $\eta$ is the strength of the Rashba spin-orbit interaction in the wire. 


\section{Ferromagnetic contact (magnetized in the z-direction)}

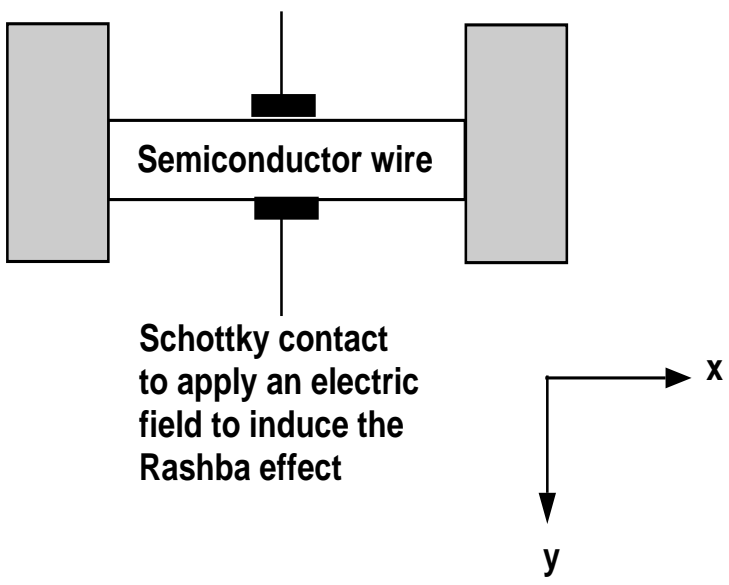

Fig. 1. Physical structure of the magnetic field sensor.

We will assume that the wire is narrow enough and the temperature is low enough that only the lowest magneto-electric subband is occupied. In that case, the Hamiltonian simplifies to [3]

$$
H=\hbar^{2} k_{x}^{2} /\left(2 m^{*}\right)+E_{0}-\beta \sigma_{x}+\eta k_{x} \sigma_{z}
$$

where $E_{0}$ is the energy of the lowest magneto-electric subband and $\beta=$ $g \mu_{B} B / 2$

Diagonalizing this Hamiltonian in a truncated Hilbert space spanning the two spin resolved states in the lowest subband yields the eigenenergies

$$
E_{ \pm}=\frac{\hbar^{2} k_{x}^{2}}{2 m^{*}}+E_{0} \pm \sqrt{\left(\eta k_{x}\right)^{2}+\beta^{2}}
$$


and the corresponding eigenstates

$$
\Psi_{+}(B, x)=\left[\begin{array}{c}
\cos \left(\theta_{k_{x}}\right) \\
\sin \left(\theta_{k_{x}}\right)
\end{array}\right] e^{i k_{x} x} \quad \Psi_{-}(B, x)=\left[\begin{array}{c}
\sin \left(\theta_{k_{x}}\right) \\
-\cos \left(\theta_{k_{x}}\right)
\end{array}\right] e^{i k_{x} x}
$$

where $\theta_{k_{x}}=-(1 / 2) \arctan \left[\beta / \eta k_{x}\right]$.

Note that if the magnetic flux density $B=0$, so that $\beta=0$, then the energy dispersion relations given in Equation (3) are parabolic, but more importantly, the eigenspinors given in Equation (4) are independent of $k_{x}$ because $\theta_{k_{x}}$ becomes independent of $k_{x}$. In fact, the eigenspinors become $[1,0]$ and $[0,1]$, which are +z-polarized and -z-polarized states. Therefore, with $B=$ 0, each of the spin resolved subbands will have a definite spin quantization axis (+z-polarized and -z-polarized). Furthermore, these quantization axes are anti-parallel since the eigenspinors are orthogonal. As a result, there are no two states in the two spin-resolved subbands that can be coupled by any nonmagnetic scatterer, be it an impurity or a phonon, or anything else. Hence, if a carrier is injected into the wire with its spin either + z-polarized or -z-polarized, then the spin will not relax (or flip) no matter how frequently energy and momentum relaxing scattering events take place. The Elliott-Yafet mechanism of spin relaxation [4] will also be completely suppressed since the eigenspinors are momentum independent. Furthermore, there is no D'yakonov-Perel' spin relaxation in a quantum wire if only a single subband is occupied [5]. Therefore, spin transport will be ballistic when $B=0$, even if charge transport is not. In other words, the spin relaxation length would have been infinite were it not for such effects as hyperfine interaction with nuclear spins which can relax electron spin. Such relaxation can be made virtually non-existent by ap- 
propriate choice of (isotopically pure) materials. Even in materials that have isotopes with strong nuclear spin, the spin relaxation rate due to hyperfine interaction is very small.

Now consider the situation when the magnetic field is non-zero $(\beta \neq 0)$. Then the eigenspinors given by Equation (4) are wavevector dependent. In this case, neither subband has a definite spin quantization axis since the spin state in either subband depends on the wavevector. Consequently, it is always possible to find two states in the two subbands with non-orthogonal spins. Any nonmagnetic scatterer (impurity, phonon, etc.) can then couple these two states and cause a spin-relaxing scattering event. In this case, no matter what spin eigenstate the carrier is injected in, spin transport is non-ballistic. That is to say, the spin relaxation length is much shorter compared to the case when there is no magnetic field. Since phonon scattering can flip spin in the presence of a magnetic field, and phonon scattering is quite strong in quantum wires (because of the van Hove singularity in the density of states) [6], the spin relaxation length can be rather small in the presence of a magnetic field. Therefore, a magnetic field drastically reduces the spin relaxation length. This is the basis of the magnetic field sensor.

The way the sensor works is as follows. Using a ferromagnetic spin injector contact magnetized in the +z-direction, we will inject carriers into the wire with +z-polarized spins. The ferromagnetic contact results in a magnetic field directed mostly in the z-direction, but also with some small fringing component along the x-direction. The z-directed component of the field does not matter, since it does not extend along the wire, which is in the x-direction. However, any x-directed component will matter. Fortunately, the fringing field decays very quickly and if the wire is long enough, we can neglect the effect 
of the fringing field. Therefore, in the absence of any external x-directed magnetic field, an injected electron will arrive with its spin polarization practically intact at the other end where another ferromagnetic contact (magnetized in the + z-direction) is placed. This second contact will then transmit the carrier completely and the spin polarized current will be high so that the device resistance will be low. However, if there is an external x-directed magnetic field, then the injected spin is no longer an eigenstate and therefore can flip in the channel [8] and arrive at the second contact with arbitrary polarization. In fact, if the wire is long enough (much longer than the spin relaxation length), then the spin polarization of the current arriving at the second contact would have essentially decayed to zero, so that there is equal probability of an electron being transmitted or reflected. In this case, the device conductance can decrease by $\sim 50 \%$ compared to the case when there is no magnetic field. This of course assumes that every carrier was initially injected with its spin completely polarized in the +z-direction. In other words, the spin injection efficiency is $100 \%$. A more realistic scenario is to assume, say, a $32 \%$ injection efficiency since it has already been demonstrated in an Fe/GaAs heterostructure [9]. For $32 \%$ injection efficiency, the conductance will decrease by $24 \%$ in a magnetic field. We will err on the side of caution and assume conservatively that the device conductance will decrease by only $10 \%$ in a magnetic field. A recent self-consistent drift diffusion simulation, carried out for a similar type of device, has shown that the conductance decreases by $20-30 \%$ because of spin flip scatterings [10].

At this point, we mention that the idea of changing device resistance by modulating spin flip scattering was the basis of a recently proposed "spin field effect transistor" [11]. Unfortunately, the small conductance modulation achievable 
by this technique (maximum 50\%) is insufficient for a "transistor" which is an active device requiring a conductance modulation by three orders of magnitude to be useful. In contrast, the device proposed here is a passive sensor that does not have the stringent requirements of a transistor and, as we show below, a $10 \%$ conductance modulation is adequate to provide excellent sensitivity.

Let us now estimate how much magnetic field can decrease the device conductance by the assumed $10 \%$. Roughly speaking, this could correspond to the situation

$$
\frac{\beta}{\eta k_{F}} \geq 0.1
$$

where $k_{F}$ is the Fermi wavevector in the channel. For a linear carrier concentration of $5 \times 10^{4} / \mathrm{cm}, k_{F} \approx 8 \times 10^{6} / \mathrm{m}$. The measured value of $\eta$ in materials such as InAs is of the order of $10^{-11} \mathrm{eV}-\mathrm{m}$ [7]. For most materials (with weaker Rashba effect), the value of $\eta$ may be two orders of magnitude smaller. Actually, this value can be tuned with an external electric field. Therefore, it is reasonable to estimate that $\eta k_{F} \sim 1 \mu \mathrm{eV}$. The magnetic field that can cause the $10 \%$ decrease in the conductance of the device is then found from Equation (5) to be $\sim 10$ Oe, if we assume $|g| \approx 15$.

We now carry out a standard sensitivity analysis following ref. [12]. The rms noise current for a single wire is taken to be the Johnson noise current given by $I_{n}=\sqrt{4 k T G \Delta f}=C \sqrt{G}$, where $k$ is the Boltzmann constant, $T$ is the absolute temperature, $G$ is the device conductance, $\Delta f$ is the noise bandwidth, and $C$ $=\sqrt{4 k T \Delta f}$. In reality, the noise current can be suppressed in a quantum wire by two orders of magnitude because of phonon confinement [6]. Therefore, $I_{n}$ $=C \sqrt{G} \zeta$, where $\zeta$ is the noise suppression factor which is about 0.01 . There 
may be also other sources of noise, such as $1 /$ f noise, but this too is suppressed in quantum wires. $1 / \mathrm{f}$ noise can be reduced by operating the sensor under an ac bias with high frequency.

The change in current through a wire in a magnetic field $H$ is the signal current $I_{s}$ and is expressed as $S H$, where $S$ is the sensitivity. We will assume that the current drops linearly in a magnetic field, so that $S$ is independent of $H$. In reality, the current is more likely to drop superlinearly with magnetic field so that $S$ will be larger at smaller magnetic field. This is favorable for detecting small magnetic fields, but since we intend to remain conservative, we will assume that $S$ is independent of magnetic field. Therefore, the signal to noise ratio for a single wire is

$$
(S: N)_{1}=\frac{S H}{I_{n}}=\frac{S H}{C \zeta \sqrt{G}}
$$

The noise current of $N$ wires in parallel is $C \sqrt{N G} \zeta$, whereas the signal current is $N S H$. Therefore, the signal-to-noise ratio of $N$ wires in parallel is $\sqrt{N}$ times that of a single wire.

For a carrier concentration of $5 \times 10^{4} / \mathrm{cm}$ as we have assumed, and for a mobility of only $25 \mathrm{~cm}^{2} / \mathrm{V}$-sec (at $4.2 \mathrm{~K}$ ), a single wire of length $10 \mu \mathrm{m}$ has a conductance $G \approx 2 \times 10^{-10}$ Siemens. At a $100 \mathrm{mV}$ bias, the current through a single wire is therefore $20 \mathrm{pA}$. Consequently, a $10 \%$ change in conductance produces a change of current by $2 \mathrm{pA}$. Since the $10 \%$ change in conductance is produced at 10 Oe, the sensitivity $S=0.2 \mathrm{pA} / \mathrm{Oe}$. At $1 \mathrm{fT}\left(=10^{-11} \mathrm{Oe}\right)$, the signal current $I_{s}$ for a single wire $=S H=2 \times 10^{-24}$ A, whereas the noise current at a temperature of $4.2 \mathrm{~K}$ is $2 \times 10^{-18} \mathrm{~A} / \sqrt{H z}$. Therefore the signal to noise ratio for a single wire is $10^{-6}: 1 / \sqrt{H z}$ and that for a parallel array 
of $10^{12}$ wires is $1: 1 / \sqrt{H z}$. Consequently, the optimum sensitivity is about 1 $\mathrm{fT} / \sqrt{\mathrm{Hz}}$ at $4.2 \mathrm{~K}$. If we repeat the calculation for $300 \mathrm{~K}$ (assuming that the mobility is reduced by a factor of 100 from its value at $4.2 \mathrm{~K}$ ), the optimum sensitivity is about $80 \mathrm{fT} / \sqrt{H z}$.

We now calculate the power consumption of the sensor. The power dissipated in a single wire is $20 \mathrm{pA} \times 100 \mathrm{mV}=2 \mathrm{pW}$ and for an array of $10^{12}$ wires, the total power dissipation is $2 \mathrm{~W}$ at $4.2 \mathrm{~K}$. At room temperature, the conductance of each wire is reduced by approximately a factor of 100 because of mobility degradation, so that the power dissipation is reduced to $20 \mathrm{~mW}$ for the array. Therefore, the sensor we have designed is a low power sensor.

It should be obvious from the foregoing analysis that we can increase the sensitivity, without concomitantly increasing power dissipation, if we decrease the carrier concentration in the wire, but increase the mobility or decrease the wire length, to keep the conductance the same. This could allow sub-femtoTesla field detection.

At a bias of $100 \mathrm{mV}$, the average electric field over a $10 \mu \mathrm{m}$ long wire is 100 $\mathrm{V} / \mathrm{cm}$. In the past, Monte Carlo simulation of carrier transport has shown that even at a much higher field than this, transport remains primarily single channeled in a quantum wire because of the extremely efficient acoustic phonon mediated energy relaxation [13]. Therefore, the assumption of single channeled transport is mostly valid.

Finally, one needs to identify a realistic route to fabricating an array of $10^{12}$ quantum wires. We propose using a porous alumina template technique for this purpose. The required sequence of steps is shown in Fig. 2. A thin foil of aluminum is electropolished and then anodized in $15 \%$ sulfuric acid at $25 \mathrm{~V}$ dc 
and at a temperature of $0^{\circ} \mathrm{C}$ to produce a porous alumina film containing an ordered array of pores with diameter $\sim 25 \mathrm{~nm}[14]$. A semiconductor is then selectively electrodeposited within the pores $[15,16]$ to create an array of vertically standing nanowires of $25 \mathrm{~nm}$ diameter. The density of wires is typically $10^{11} / \mathrm{cm}^{2}$, so that $10^{12}$ wires require an area of $10 \mathrm{~cm}^{2}$. Next a ferromagnetic metal is electrodeposited within the pores [17] on top of the semiconductor. The pores are slightly overfilled so that the ferromagnetic metal makes a twodimensional layer on top. This is then covered with an organic layer to provide mechanical stability during the later steps.

The aluminum foil is then dissolved in $\mathrm{HgCl}_{2}$, and the alumina barrier layer at the bottom of the pores is etched in phosphoric acid to open up the pores from the bottom. Another ferromagnet is then electrodeposited on the exposed tips of the semiconductor wires through a mask. The organic layer is then dissolved in acetone and the multilayered film is harvested and placed on top of a conducting substrate covered with silver paste. Two wires are attached to two remote diffused Schottky contacts in order to apply a transverse electric field that induces the Rashba effect. Finally wires are attached to top and bottom to provide ohmic electrical contacts. This completes the fabrication of the sensor.

In conclusion, we have proposed a highly sensitive solid-state magnetic field detector based on spin orbit interaction. Such sensors can operate at room temperature since the energy separation between subbands can exceed the room temperature thermal energy in 25-nm diameter wires. The sensitivity of these sensors could be comparable to those of superconducting quantum interference devices $[18,19,20]$ and therefore may be appropriate for magnetoencephalography where one directly senses the very small magnetic fields pro- 


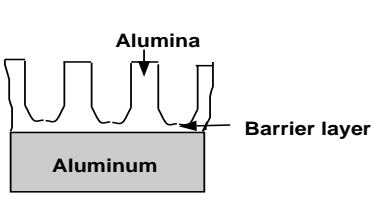

(a)

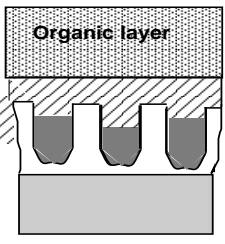

(d)

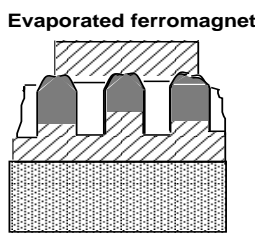

(g)

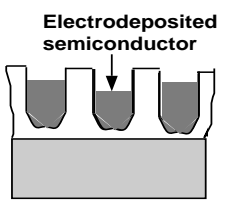

(b)

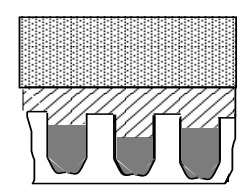

(e)

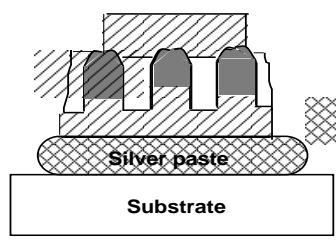

(h)

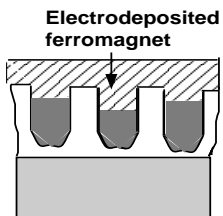

(c)

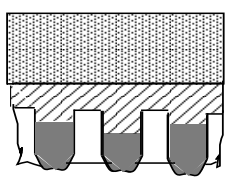

(f)

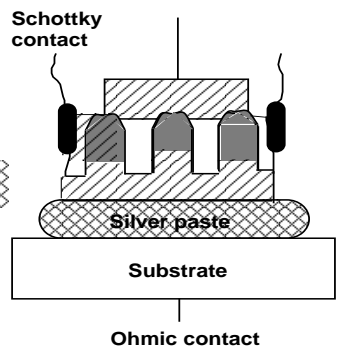

(i)

Fig. 2. Fabrication steps. (a) Creation of a porous alumina film with $25 \mathrm{~nm}$ diameter pores. This is produced by anodizing an aluminum foil at $25 \mathrm{~V}$ dc in sulfuric acid at $0^{\circ} \mathrm{C}$. (b) Electrodepositing a semiconductor selectively within the pores. (c) Electrodepositing a ferromagnetic layer on top. This could be either a metallic ferromagnet, or a semiconducting ferromagnet such as ZnMnSe. (d) Pasting a thick organic layer on top for mechanical stability of the structure. (e) Dissolving out the $\mathrm{Al}$ foil in $\mathrm{HgCl}_{2}$. (f) Etching the alumina in phosphoric acid at room temperature to expose the tips of the semiconductor wires. (g) Evaporating a ferromagnet on the tips of the semiconductor wires through a mask. (h) Dissolving the organic layer in ethanol, harvesting the film and placing it on top of a conducting substrate covered with silver paste. (i) Making Schottky contacts on the sides and ohmic contacts at top and bottom.

duced by neural activity in human brains. 
The work of S. B. is supported by the Air Force Office of Scientific Research under grant FA9550-04-1-0261. 


\section{References}

[1] G. Dresselhaus, Phys. Rev., 100, 580 (1955).

[2] E. I. Rashba, Sov. Phys. Semicond., 2, 1109 (1960); Y. A. Bychkov and E. I. Rashba, J. Phys. C, 17, 6039 (1984).

[3] S. Bandyopadhyay, S. Pramanik and M. Cahay, Superlat. Microstruct., 35, 67 (2004).

[4] R. J. Elliott, Phys. Rev., 96, 266 (1954).

[5] S. Pramanik, S. Bandyopadhyay and M. Cahay, www.arXiv.org/condmat/0403021. (Also to appear in IEEE Trans. Nanotech.).

[6] A. Svizhenko, A. Balandin, S. Bandyopadhyay and M. A. Stroscio, Phys. Rev. B, 57, 4687 (1998).

[7] J. Nitta, T. Akazaki, H. Takayanagi and T. Enoki, Phys. Rev. Lett., 78, 1335 (1997).

[8] M. Cahay and S. Bandyopadhyay, Phys. Rev. B, 69, 045303 (2004).

[9] A. T. Hanbicki, et al., Appl. Phys. Lett., 80, 1240 (2002). This experiment pertains to spin injection in bulk semiconductors. Spin injection in quantum wires may be even more efficient. In fact, our recent theoretical work shows that nearly $100 \%$ spin injection efficiency is possible at certain injection energies, even if the ferromagnetic contact has much less than $100 \%$ spin polarization (M. Cahay and S. Bandyopadhyay, unpublished).

[10] E. Shafir, M. Shen and S. Saikin, www.arXiv.org/cond-mat/0407416.

[11] J. Schliemann, J. C. Egues and D. Loss, Phys. Rev. Lett., 90, 146801 (2003); X. Cartoixá, D. Z-Y Ting and Y-C Chang, Appl. Phys. Lett., 83, 1462 (2003). 
[12] M. Tondra, J. M. Daughton, D. Wang, R. S. Beech, A. Fink and J. A. Taylor, J. Appl. Phys., 83, 6688 (1998).

[13] A. Svizhenko, S. Bandyopadhyay and M. A. Stroscio, J. Phys: Condens. Matter, 11, 3697 (1999).

[14] See, for example, K. Nielsch, J. Choi, K. Schwirn, R. B. Wehrspohn and U. Gösele, Nano Letters, 2, 677 (2002) and references therein.

[15] N. Kouklin, L. Menon, A. Z. Wong, D. W. Thompson, J. A. Woollam, P. F. Williams and S. Bandyopadhyay, Appl. Phys. Lett., 79, 4423 (2001).

[16] S. Bandyopadhyay, L. Menon, N. Kouklin, P. F. Williams and N. J. Ianno, Smart. Mater. Struct., 11, 761 (2002).

[17] L. Menon, M. Zheng, H. Zeng, S. Bandyopadhyay and D. J. Sellmyer, J. Elec. Mater., 29, 510 (2000).

[18] J. Gallop, Supercond. Sci. Technol., 16, 1575 (2003).

[19] H. J. Barthelmess, et al., IEEE Trans. Appl. Supercond., 11, 657 (2001).

[20] M. Pannetier, C. Fermon, G. Le Goff, J. Simola and E. Kerr, Science, 304, 1648 (2004). 